\title{
Multicentric Papillary Thyroid Carcinoma: Stratification for Treatment
}

\author{
Salvador J. Diaz-Cano*
}

Department of Histopathology, King's College Hospital and King's Health Partners, London, United Kingdom

\begin{abstract}
Tumor stage is the main prognosticator of differentiated thyroid carcinomas, but it needs further stratification for multicentric neoplasms (most frequently papillary thyroid carcinomas, PTC), which frequently present as foci smaller than $1 \mathrm{~cm}$ and are considered by default as high-risk tumors. Not all cases of multicentric PTC should be considered of high risk and they should be stratified to avoid unnecessary radioiodine ablation in selected cases. Currently, there are known variables for this stratification: size and number of tumor foci, clonal patterns, and specific markers of aggressive behaviour (infiltrative growth, BRAF mutation). In a near future, the identification of a gene expression pattern associated with a higher risk of recurrence would allow us to focus more aggressive treatment appropriately.
\end{abstract}

Keywords: Thyroid; Papillary carcinoma; Multicentric; Treatment stratification

\section{Abbreviations: miRNA: Micro RNA; PTC: Papillary Thyroid Carcinoma}

\section{Short Commentary on Early Multicentric PTC}

The treatment of papillary thyroid carcinoma (PTC) has significantly changed recently and it is mainly based on surgery and radioiodine ablation, the latter for high-risk patients only [1-4]. However, this treatment is currently subjected to extensive review especially for low risk carcinomas. Two main factors have contributed to this review: the increased incidence of secondary neoplasms after radioiodine treatment and the efficiency demonstrated by low dose treatment [4,5]. Cancer treatment selection depends on patient stratification according to risk for a given tumor, but it is particularly challenging for patients with low-risk thyroid cancers because of the slow growth of such tumors. Tumor stage is the main prognosticator of differentiated thyroid carcinomas, but it needs further stratification for multicentric neoplasms (most frequently PTC), which frequently present as foci smaller than $1 \mathrm{~cm}$ and are considered by default as highrisk tumors. Microscopic PTCs $(<1.0 \mathrm{~cm})$ are considered a subset of PTCs that behave more benign. They follow an indolent course and carry an excellent prognosis. Distant metastases and mortality rates were reported to be less than $0.5 \%$ [6]. However, some authors suggest that there exists a subgroup of microscopic PTCs that can be aggressive, requiring therapeutic management similar to larger tumors [7]. Unfortunately, within this set of patients, prognostic factors have not been well defined. However, in recent years some specific markers for aggressiveness were identified, including sizes larger than $5 \mathrm{~mm}$, multifocality, tumor extension beyond the parenchyma, lymph node involvement, tumor non-incidentally discovered, and the extent of primary surgery [7-10].

Currently, both biologic and pathologic variables provide a reliable basis for risk stratification of early stage thyroid cancer. Intratumor heterogeneity is at the foundation of tumor progression and it correlates with the tumor volume and multicentricity [11]: the potential to metastasize increase directly with primary size (the main element for T staging), and multicentric neoplasms (expressed by (m) in the $\mathrm{T}$ classification). These two key elements are the main general prognosticators used to plan any adjuvant radioiodine treatment. Although multicentric PTCs are not stratified further considering that the presence of multicentricity provides a risk for all patients, no statistical difference in cancer mortality has been observed between the ablated and nonablated groups of patients with multicentric PTC [12], most likely reflecting the lack of selection criteria.

Multicentricity is considered an expression of tumor heterogeneity, the main driver of tumor progression by increasing the number of different clones and therefore the probability of sub-clone development with additional acquired capabilities [11]. Multicentric PTC is more frequently detected due to improved diagnostic methods. More than $70 \%$ of microscopic PTCs are diagnosed incidentally (in specimens of the thyroid removed for benign thyroid disease) and it is responsible for the $2.4 \mathrm{x}$ increase in incidence of differentiated thyroid carcinoma [13]. Multiple foci have been reported in approximately $7-56 \%$ of microscopic PTCs $[6,14]$. A number of clinical studies showed that patients with $\geq$ two foci had higher recurrence rate and cancer mortality than those with unifocal PTMCs [6,12]. Moreover, multifocality is an independent risk factor for metastases [15]. It was demonstrated that cases with positive lymph nodes had a higher risk of recurrence [13]. Hence, multifocal PTMCs have been considered to have a poor prognosis requiring radioiodine ablation and close surveillance within the first year. Patients with multicentric microscopic PTC need to be treated as highrisk patients, which partially explain the increase in the proportion of patients receiving radioactive iodine $[5,16]$. The recurrence rate increases significantly in cases with $\geq 5$ foci, which frequently undergo ${ }^{131} \mathrm{I}$ ablation [12]. As all foci are governed by the volume growth criteria expressed below, the clinical significance given to each focus must be linked to its size and a demonstrable evidence of different cellular progenitor. Therefore, the stratification of patients with multicentric tumors would require considering both the size of each focus, the number of foci, and a sensible application of clonality tests (such as X-chromosome inactivation and fractional allelic loss assays) [17]. An appropriate use of these markers serves to

*Corresponding author: Salvador J. Diaz-Cano, King's College Hospital, Dept of Histopathology, Denmark Hill, London, Greater London, SE5 9RS, United Kingdom, Tel: +44 20 3299-3041 Fax: +44 20 3299-3670; E-mail: sdiaz-cano@nhs.net

Received December 18, 2013; Accepted March 29, 2014; Published March 31 2014

Citation: Diaz-Cano SJ (2014) Multicentric Papillary Thyroid Carcinoma: Stratification for Treatment. J Cytol Histol 5: 238. doi:10.4172/21577099.1000238

Copyright: $\odot 2014$ Diaz-Cano SJ. This is an open-access article distributed under the terms of the Creative Commons Attribution License, which permits unrestricted use, distribution, and reproduction in any medium, provided the original author and source are credited. 
separate clonal origin from clonal expansion (sub-clones development contributing to heterogeneity and progression). As founding mutations have not been properly established in many tumors, clonality assays carry this caveat. However it is only through fractional allelic loss and mutation burden assessments that the relationships within the subclones become established. Tumors showing a common progenitor (monoclonal neoplasms) most likely represent intrathyroidal extension and frequently reveal good prognosis, even when associated with lymph node metastasis, provided that no extrathyroidal extension is identified. Multicentric PTC originates usually from neoplastic transformation and subsequent intrathyroidal spread of a single malignant progenitor clone. Bilateral PTCs frequently show the same pattern of inactivation (80\%), suggesting that bilateral, recurrent, and metastatic papillary thyroid carcinomas often arise from a single clone and that intrathyroidal metastasis may play an important role in the development of bilateral tumors [18]. Clonal progression and morphotype differentiation occur through progressive acquisition of genetic alterations subsequent to the initial intra-glandular spread. The combination of both tumor focus size and clonality gives the most biologically meaningful approach to stratify patients.

In the tumor volume stratification, the stages are intimately associated with the size of the primary neoplasm: tumors bigger than $1.0 \mathrm{~cm}$ reveal an exponential volume growth with good linear correlation $\left(\mathrm{R}^{2}=0.96463\right)$ and slopes over $3(14.582$ for tumors $2-4 \mathrm{~cm}$, 3.646 for neoplasms $1-2 \mathrm{~cm}$ ); tumors $0.5-1 \mathrm{~cm}$ maintains the linear correlation, but showing much lower slope $\left(0.911, \mathrm{R}^{2}=0.96463\right)$, while tumors smaller than $0.5 \mathrm{~cm}$ reveal a partial loss of the linear correlation $\left(\mathrm{R}^{2}<0.9\right)$ and a significantly lower slope $(0.202$ for tumors $0.2-0.5 \mathrm{~cm}$, 0.021 for tumors $0.02-0.2 \mathrm{~cm}$, and 0.0002 for tumors smaller than $0.02 \mathrm{~cm}$ ) (Figure 1). These figures represent a slight overestimation of the tumor growth because they are based on the assumption of pure tumor cell proliferation, replication of tumor cells in two descendent cells, and a default spherical tumor volume. The tumor cell burden is going to be smaller than the initially predicted due to the heterotypic neoplasm biology: the presence of interstitial host cells (inflammatory cells, endothelial cells, stromal cells, which can have positive or negative effect on tumor growth) and non-solid tumor cell architecture (follicular of papillary in thyroid neoplasms). These two factors will reduce the number of tumor cells estimated per volume, therefore decreasing the cellular pool on which tumor progression can develop. Based on tumor size alone, its impact on tumor volume would therefore be: clinically relevant for tumor foci $0.5-1.0 \mathrm{~cm}(1: 1)$, limited for tumor foci $0.2-0.5 \mathrm{~cm}(1: 5)$, very limited for tumor foci $0.02-0.2 \mathrm{~cm}(2: 100)$, and almost absent for tumor foci smaller than $0.02 \mathrm{~cm}$ (2:10000). These considerations of tumor size must be applied to each focus in case of multicentric neoplasms, including for the risk assessment only those foci clinically relevant according to its size and provided that they do not show other adverse features. This size assessment in the primary tumor also shares the approach with the evaluation of lymph node metastasis, which distinguishes metastasis $(>2 \mathrm{~mm})$, micrometastasis $(0.2-2 \mathrm{~mm})$ and isolated tumor cells $(<0.2 \mathrm{~mm},<200$ cells $)$.

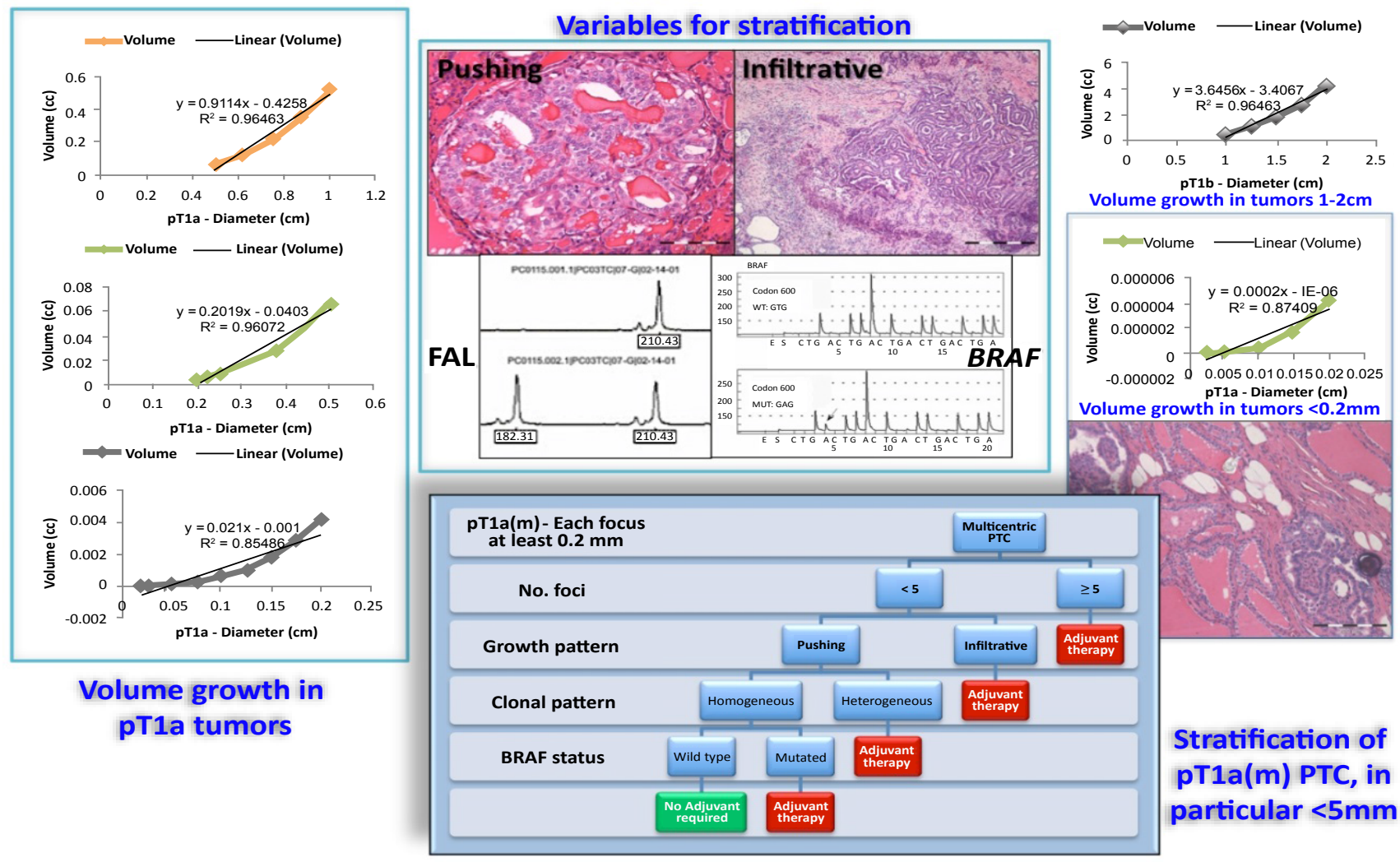

Figure 1: Tumor focus size approach from the upper range clearly separates four scenarios for pT1a tumors: (a) tumors $0.5-1.0 \mathrm{~cm}$ reveal almost $1: 1$ increase of tumor volume per diameter unit, (b) tumors $0.2-0.5 \mathrm{~cm}$ show about one fifth growth of tumor volume per diameter unit, (c) tumors $0.02-0.2 \mathrm{~cm}$ increase around 2 hundredth of tumor volume per diameter unit, and (d) tumors smaller than $0.02 \mathrm{~cm}$ expand about 2 ten-thousandth the tumor volume per diameter unit. This volume growth has to be coupled with number of foci, histological growth pattern (pushing vs. infiltrative), clonality pattern (homogeneous vs. heterogeneous) and simple genotyping (BRAF status) to determine the need of adjuvant therapy in multicentric PTC less than $1 \mathrm{~cm}(\mathrm{pT} 1 \mathrm{a}(\mathrm{m}))$, but with particular emphasis for tumor smaller than $5 \mathrm{~mm}$ that are being more frequently detected due to more exhaustive histological examination. FAL $=$ Fractional allelic loss. 
Citation: Diaz-Cano SJ (2014) Multicentric Papillary Thyroid Carcinoma: Stratification for Treatment. J Cytol Histol 5: 238. doi:10.4172/21577099.1000238

Apart from generic variables (size and clonality), there are a number of specific known criteria of high clinical risk for metastasis. These factors are normally referred to as markers of aggressive behaviour and they must be considered for patient stratification. Established markers of aggressive PTC behaviour include both morphological and genetic variables that need to be evaluated in each PTC focus: histologic growth patterns (such as sclerosing, infiltrative or tall cell variants) and $B R A F$ mutations.

Only rarely tumors less than $0.5 \mathrm{~cm}$ have associated metastases. However, despite the presence of lymph node metastases, the incidence of death from lesions of this size range is extremely rare, frequently associated with other risk factors, such as the presence of aneuploid cell populations in the tumor, sclerosing infiltrative architecture and peripheral location within the gland where it can invades perithyroidal soft tissues and lymphatics [19]. Cyclin D1 staining of PTC is very useful for identifying the intrathyroidal spreading or multifocality of the tumors [20]. In monoclonal BRAF V600E-positive multicentric PTCs, BRAF V600E is not always present in all tumor foci, indicating that other tumor-genetic factors in the primary progenitor clone can also trigger PTC neoplastic transformation [2]. Micro-RNA (miRNA) profiles can distinguish tumors containing the BRAF mutation from the other tumor types, and to differentiate between the more aggressive histological patterns linked to various processes involved in tumor growth and proliferation. Hierarchical clustering analysis of miRNA expression suggests that both discrete areas do not evolve from clonal expansion of tumor cells and independent mutational events can occur simultaneously within a tumor to enhance cancer progression in geographical micro-environments within a tumor [21].

Multicentric PTC should also be stratified and not all cases considered of high risk and, therefore, requiring radioiodine ablation. Currently, there are known variables for this stratification: size and number of tumor foci, clonal patterns, and specific markers of aggressive behaviour (infiltrative growth, $B R A F$ mutation). In a near future, the identification of a gene expression pattern associated with a higher risk of recurrence would allow us to focus more aggressive treatment appropriately.

\section{References}

1. Alexander EK, Larsen PR (2012) Radioiodine for thyroid cancer--is less more? N Engl J Med 366: 1732-1733.

2. Jovanovic L, Delahunt B, Mclver B, Eberhardt NL, Grebe SK (2008) Most multifocal papillary thyroid carcinomas acquire genetic and morphotype diversity through subclonal evolution following the intra-glandular spread of the initial neoplastic clone. J Pathol 215: 145-154.

3. Mallick U, Harmer C, Yap B, Wadsley J, Clarke S, et al. (2012) Ablation with low-dose radioiodine and thyrotropin alfa in thyroid cancer. $\mathrm{N}$ Engl $\mathrm{J}$ Med 366: 1674-1685.

4. Schlumberger M, Catargi B, Borget I, Deandreis D, Zerdoud S, et al. (2012) Strategies of radioiodine ablation in patients with low-risk thyroid cancer. N Engl J Med 366: 1663-1673.

5. Haymart MR, Banerjee M, Stewart AK, Koenig RJ, Birkmeyer JD, et al. (2011) Use of radioactive iodine for thyroid cancer. JAMA 306: 721-728.
6. Hay ID, Hutchinson ME, Gonzalez-Losada T, Mclver B, Reinalda ME, et al. (2008) Papillary thyroid microcarcinoma: a study of 900 cases observed in a 60-year period. Surgery 144: 980-987.

7. Page C, Biet A, Boute P, Cuvelier P, Strunski V (2009) 'Aggressive papillary' thyroid microcarcinoma. Eur Arch Otorhinolaryngol 266: 1959-1963.

8. Küçük NO, Tari P, Tokmak E, Aras G (2007) Treatment for microcarcinoma of the thyroid--clinical experience. Clin Nucl Med 32: 279-281.

9. Lee X, Gao M, Ji Y, Yu Y, Feng Y, et al. (2009) Analysis of differential BRAF(V600E) mutational status in high aggressive papillary thyroid microcarcinoma. Ann Surg Oncol 16: 240-245

10. Mercante G, Frasoldati A, Pedroni C, Formisano D, Renna L, et al. (2009) Prognostic factors affecting neck lymph node recurrence and distant metastasis in papillary microcarcinoma of the thyroid: results of a study in 445 patients. Thyroid 19: 707-716.

11. Diaz-Cano SJ (2012) Tumor heterogeneity: mechanisms and bases for a reliable application of molecular marker design. Int J Mol Sci 13: 1951-2011.

12. Lin JD, Chao TC, Hsueh C, Kuo SF (2009) High recurrent rate of multicentric papillary thyroid carcinoma. Ann Surg Oncol 16: 2609-2616.

13. Chow SM, Law SC, Chan JK, Au SK, Yau S, et al. (2003) Papillary microcarcinoma of the thyroid-Prognostic significance of lymph node metastasis and multifocality. Cancer 98: 31-40.

14. Dietlein M, Luyken WA, Schicha H, Larena-Avellaneda A (2005) Incidental multifocal papillary microcarcinomas of the thyroid: is subtotal thyroidectomy combined with radioiodine ablation enough? Nucl Med Commun 26: 3-8.

15. GÃ/4lben K, BerberoÄŸlu U, Celen O, Mersin HH (2008) Incidental papillary microcarcinoma of the thyroid--factors affecting lymph node metastasis. Langenbecks Arch Surg 393: 25-29.

16. Hughes DT, Haymart MR, Miller BS, Gauger PG, Doherty GM (2011) The most commonly occurring papillary thyroid cancer in the United States is now a microcarcinoma in a patient older than 45 years. Thyroid 21: 231-236.

17. Diaz-Cano SJ, Blanes A, Wolfe HJ (2001) PCR techniques for clonality assays. Diagn Mol Pathol 10: 24-33.

18. Wang W, Wang H, Teng X, Wang H, Mao C, et al. (2010) Clonal analysis of bilateral, recurrent, and metastatic papillary thyroid carcinomas. Hum Pathol 41: 1299-1309.

19. Strate SM, Lee EL, Childers JH (1984) Occult papillary carcinoma of the thyroid with distant metastases. Cancer 54: 1093-1100.

20. Jung CK, Kang YG, Bae JS, Lim DJ, Choi YJ, et al. (2010) Unique patterns of tumor growth related with the risk of lymph node metastasis in papillary thyroid carcinoma. Mod Pathol 23: 1201-1208.

21. Aherne ST, Smyth PC, Flavin RJ, Russell SM, Denning KM, et al. (2008) Geographical mapping of a multifocal thyroid tumour using genetic alteration analysis \& miRNA profiling. Mol Cancer 7: 89. 